\title{
Self-consistent analysis of electric field effects on Si $\delta$-doped GaAs
}

\author{
José A Cuestał, Angel Sánchez $\nmid$ and \\ Francisco Dominguez-Adame $\ddagger$
}

\author{
† Escuela Politécnica Superior, Universidad Carlos III de Madrid, C./ Butarque 15, \\ E-28911 Leganés, Madrid, Spain \\ $\ddagger$ Departamento de Física de Materiales, Facultad de Físicas, Universidad \\ Complutense, E-28040 Madrid, Spain
}

\begin{abstract}
We theoretically study the subband structure of single Si $\delta$-doped GaAs inserted in a quantum well and subject to an electric field applied along the growth direction. We use an efficient self-consistent procedure to solve simultaneously the Schrödinger and Poisson equations for different values of electric field and temperature. We thus find the confining potential, the subband energies and their corresponding envelope functions, the subband occupations, and the oscillator strength of intersubband transitions. Opposite to what is usually the case when dealing with the quantum-confined Stark effect in ordinary quantum wells, we observe an abrupt drop of the energy levels whenever the external field reaches a certain value. This critical value of the field is seen to depend only slightly on temperature. The rapid change in the energy levels is accompanied by the appearance of a secondary well in the confining potential and a strong decrease of the oscillator strength between the two lowest subbands. These results open the possibility to design devices for use as optical filters controlled by an applied electric field.
\end{abstract}

\section{Introduction}

Recent advances in epitaxial growth techniques, such as molecular beam epitaxy (MBE). make it possible to fabricate high quality $\mathrm{Si} \delta$-doped layers in GaAs. In these systems, a slab of $\mathrm{Si}$ atoms localized within a few monolayers supplies electrons and gives rise to quantum confinement of carriers. By this means, a two-dimensional electron gas can be realized by planar doping of $\mathrm{GaAs}$ at high donor concentration [1]. Devices based on $\delta$-doped heterostructures are currently under extensive investigation for high-speed electronic and optoelectronic applications (see, e.g., [2] for some examples of the practical advantages of $\delta$-doping). Hence there is great interest in a good understanding of $\mathrm{Si} \delta$-doped $\mathrm{GaAs}$ as a representative example of those devices.

Theoretical studies of the above systems usually neglect possible effects of disorder due to the random distribution of impurities in order to simplify the analysis. Indeed, currently available techniques aliow for an optimai control of the growing heterostructure, thus justifying the assumption that the ionized impurity atoms are homogeneously distributed inside the $\delta$-doped layers. This approximation has recently been shown to be correct in the high-density linnit [3]. A number of researches have considered this limit within different approaches, like the Thomas-Fermi [4], local density approximation (LDA) [5] and Hartree methods [6]. These previous works show that in the absence of external fields the Thomas-Fermi semiclassical approach is equivalent to a self-consistent formulation over a wide range of doping concentrations [4]. The effects of applied electric field have recently been considered in the case of single and periodically Si $\delta$-doped GaAs $[7,8]$ by using a generalized ThomasFermi formalism. In this framework one first computes the one-electron potential in the absence of electric field and then tilts it to account for the electric potential. It has thus been found that the well-known Stark ladders, already observed in quantum-well based superlattices [9], should also be clearly revealed in periodically $\mathrm{Si} \delta$-doped GaAs. Nevertheless, this ad hoc procedure might be incapable of describing all the phenomenology arising in $\delta$-doped heterostructures: a fully self-consistent approach may reveal interesting issues about the behaviour of actual structures under bias voltage not accounted for within simpler approaches.

As an example of new phenomenology found by selfconsistent procedures, we mention that, in superlattice-like systems, the Hartree potential from electrons of different wells partly screens the effect of the electric field, leading to the formation of electric field domains. The physical existence of electric field domains is firmly established in GaAs-Ga $a_{-x} \mathrm{Al}_{x}$ As [10] supperlattices (see [11] and references therein). To our knowledge, however, there have been no reports in the literature about field domains in $\delta$-doped GaAs superlattices. Note that in this case 
there are a number of free carriers in the structure and, as a consequence, it is possible for the electric field to break up into two or more regions with different field strengths, i.e. electric field domains. Similarly, it would not be strange to observe other unexpected phenomena when a more complete theoretical analysis of $\delta$-doped systems under applied fields is carried out. It is therefore clear that a careful self-consistent analysis of this kind of device is necessary to make sure what their properties as well as their possible applications are.

The work we report on in this paper is a first step in the aforementioned direction. We have concerned ourselves with the self-consistent study of a single Si $\delta$ doped layer in GaAs under an applied electric field. The aim of addressing this question is twofold. On the one hand, we intend to implement and improve an efficient self-consistent procedure to analyse this simple structure, thus developing the necessary skills to tackle the full superlattice problem. It is important to realize that a selfconsistent study of a complex heterostructure requires a well developed technique to prevent expensive calculations. On the other hand, we believe that only after achieving a thorough knowledge of the phenomena appearing in a single layer will it be possible to proceed further, in order to assess the electronic structure of periodically $\mathrm{Si} \delta$-doped GaAs superlattices. In addition, if our work showed new features of the single layer problem, we could foresee the subsequent, unexpected phenomena which would be likely to arise in the superlattice, as well as estimate the range of parameters for which they might take place. Therefore, a complete understanding of charge distribution and subband energy dependence on the applied electric field in a single Si $\delta$-doped GaAs layer is necessary. With this double goal in mind, we undertook the study of the quantum-confined Stark effect in these structures by considering a $\delta$-doping layer in a quantum well.

The paper is organized as follows. In section 2 we briefly discuss our model, in which we use a scalar Hamiltonian within the effective-mass approximation to describe the electron dynamics. The one-electron potential due to the combined action of the ionized donors in the single $\delta$-doped layer and the applied electric field is found by simultaneously solving the Schrödinger and Poisson equations. Section 3 is devoted to a summary of the numerical method we apply to obtain (i) the one-electron potential, (ii) the dependence of the subband structure upon the applied electric field, (iii) the subband occupation as a function of the field, (iv) the spatial charge distribution and ( $v$ ) the oscillator strength for intersubband transitions when the whole structure is confined between two infinitely high barriers in an applied electric field (quantum-confined Stark effect). Results and discussions are collected in section 4, and section 5 endòs the pāper with à briế recollection of results and some comments on possible physical consequences and technological applications of our results in new devices.

\section{M̄odiei}

The system we study in this work is a semiconductor structure made of a single $\mathrm{Si} \delta$-doped GaAs layer. We consider a slab of GaAs of thickness $L$ confined between two infinitely high barriers with a $\mathrm{Si} \delta$-doping layer embedded in its centre. We assume that the doping layer consists of a continuous slab of thickness $d$ with $N_{D}^{+}$ ionized donors per unit area. In what follows we neglect the unintentional p-type background doping appearing in most MBE-grown samples. This is not a serious limitation since actual techniques can keep this doping level very low (less than $10^{14} \mathrm{~cm}^{-3}$ acceptors). For such a low residual doping, the well shape below the Fermi level $E_{F}$ is almost insensitive to the background acceptors [12].

We assume the validity of the effective-mass approximation and take an isotropic and parabolic conduction band in the growth direction. This approximation usually works fine in GaAs, except at very high electric fields, when $\Gamma-X$ mixing induced by the field occurs [13]. Kane's parameter $\left(E_{p} \simeq 23 \mathrm{meV}\right)$ and the conductítion-band modulation are much smaller than the bandgap in GaAs, and the coupling between host bands is small, so that a scalar Hamiltonian suffices to properly describe the electronic conduction-band states in $\delta$-doped GaAs [14]. In the envelope function approach, the electronic wavefunction corresponding to the $j$ th subband may be factorized as follows:

$$
\psi_{j}(r)=\frac{1}{\sqrt{S}} \exp \left(\mathrm{i} k_{\perp} \cdot r_{\perp}\right) \psi_{j}(z)
$$

where $k_{\perp}$ and $r_{\perp}$ are the in-plane wavevector and spatial coordinates, respectively. Here $S$ is the area of the Iayer. The subband energy follows the parabolic dispersion law $E_{j}+\hbar^{2} k_{\perp}^{2} / 2 m^{*}, m^{*}$ being the electron effective mass at the bottom of the conduction band ( $\Gamma$ valley). The quantized energy levels $E_{j}$ and their corresponding envelope functions $\psi_{j}(z)$ satisfy the following Schrödinger-like equation:

$$
\left(-\frac{\hbar^{2}}{2 m^{*}} \frac{\mathrm{d}^{2}}{\mathrm{~d} z^{2}}+V(z)\right) \psi_{j}(z)=E_{j} \psi_{j}(z) .
$$

The presence of infinite barriers implies that the envelope functions vanish at $z= \pm L / 2$.

The-one-electron potential splits into four different contributions:

$$
V(z)=V_{b}(z)+e F z+V_{x c}(z)+V_{H}(z) .
$$

Here $V_{b}(z)$ is the built-in potential due to the infinite barriers. Therefore $V_{b}(z)=0$ for $|z|<L / 2$ and becomes infinite otherwise. $F$ is the strength of the applied electric field. $V_{x c}(z)$ is the local exchange-correlation energy calculated in the LDA approximation using the HedinLundqvist parametrization [15],

$$
V_{x c}(z)=-\frac{R y^{*}}{10.5 \pi \alpha r}[1+0.7734 r \ln (1+1 / r)]
$$

where we have defined

$$
\alpha=(4 / 9 \pi)^{1 / 3} \quad \text { and } \quad r=\frac{1}{21}\left(\frac{4}{3} \pi a^{* 3} n(z)\right)^{-1 / 3}
$$

$n(\bar{z})$ is the electronon densitity, añd $R \bar{y}^{*}=e^{2} / 8 \pi \epsilon_{0} k a^{*}$ and $a^{*}=4 \pi \epsilon_{0} K \hbar^{2} / m^{*} e^{2}$ are respectively the effective Rydberg and the effective Bohr radius. The local dielectric constant $\kappa$ is assumed not to depend on the spatial coordinate in the 
whole structure. The Hartree potential $V_{H}(z)$ is obtained by solving the one-dimensional Poisson equation

$$
\frac{\mathrm{d}^{2} V_{H}(z)}{\mathrm{d} z^{2}}=\frac{e}{\epsilon_{0} K}\left[N_{D}^{3 D}(z)-n(z)\right]
$$

along with the boundary conditions $V_{H}(z=-L / 2)=$ $V_{H}(z=+L / 2)=0$, where $N_{D}^{3 D}(z)=N_{D}^{+} / d$ for $|z|<d / 2$ and vanishes in other regions. The electron density can be written as

$$
n(z)=\sum_{j} n_{j}\left|\psi_{j}(z)\right|^{2}
$$

where the sum runs over the different subbands. The subband occupation $n_{j}$ is given by

$$
n_{j}=\frac{k_{B} T m^{*}}{\pi \hbar^{2}} \ln \left[\hat{1}+\exp \left(\frac{E_{F}-E_{j}}{k_{B} T}\right)\right] .
$$

This set of equations must be solved until self-consistency is reached. Then envelope functions and electron energies can be found as a function of the applied field. Once these magnitudes are computed, it is easy to obtain the charge distribution and subband occupations. In addition, regarding field-dependent intersubband transitions from a state $k$ into a state $j$, it becomes most important to determine the corresponding oscillator strength

$$
f_{j k}=\frac{2 m_{0}\left(E_{k}-E_{j}\right)}{\hbar^{2}}\left|\left\langle\psi_{k}|z| \psi_{j}\right)\right|^{2}
$$

where $m_{0}$ is the free-electron mass. Device applications of intersubband transitions require a large Stark shift with a high oscillator strength. For example, it is known that square quantum wells exhibit a weak intersubband transition [16], but it can be improved by inserting one narrow well inside a wider one $[17,18]$. Therefore it is interesting, from a technological viewpoint, to compare the oscillator strength in $\delta$-doped structures with typical values obtained in quantum wells.

\section{Numerical analysis}

\subsection{Dimensionless variables}

For convenience, we begin by introducing dimensionless variables in our problem. We can define reduced well length, $l$, doping layer thickness, $\Delta$, and position coordinate, $x$, by rescaling $L, d$ and $z$ with $a^{*}$, respectively. Furthermore, we introduce reduced energies, $\epsilon$, and potentials, $v$, by rescaling $E$ and $V$ with $R y^{*}$. On the other hand, reduced electric fields, $f$, charge densities, $\nu$, and temperatures, $\tau$, are defined by rescaling $F, n$ and $T$ respectively with their corresponding scaling factors $F_{0} \equiv R y^{*} / e a^{*} \approx 5.91 \mathrm{kV} \mathrm{cm}{ }^{-1}, n_{0} \equiv\left(a^{*}\right)^{-3} \approx 10^{18} \mathrm{~cm}^{-3}$ and $T_{0} \equiv R y^{*} / k_{B} \approx 67.7 \mathrm{~K}$. With these new variables equation (3) becomes

$$
v(x)=v_{b}(x)+f x+v_{x c}(x)+v_{H}(x)
$$

In this equation, $v_{b}(x)=0$ for $|x|<l / 2$ and is infinite otherwise. The reduced exchange potential, $v_{x c}$, is given by

$$
\begin{aligned}
& v_{x c}(x)=-\frac{1}{7}\left(\frac{2}{3 \pi^{2}}\right)\{u(x)+0.7734 \ln [1+u(x)]\} \\
& u(x) \equiv 7(36 \pi)^{1 / 3} \nu(x)^{1 / 3}
\end{aligned}
$$

and $v_{H}(x)$ is determined by solving

$$
v_{H}^{\prime \prime}(x)=8 \pi\left[v_{D}^{3 D}(x)-v(x)\right] .
$$

Here $v_{D}^{3 D}(x)=\left(a^{*}\right)^{2} N_{D}^{+} / \Delta$ for $|x|<\Delta / 2$ and 0 otherwise, and $v(x)$ is computed through

$$
\begin{aligned}
v(x) & =\sum_{j} v_{j}\left|\chi_{j}(x)\right|^{2} \\
v_{j} & =\frac{\tau}{2 \pi} \ln \left[1+\exp \left(\frac{\epsilon_{F}-\epsilon_{j}}{\tau}\right)\right]
\end{aligned}
$$

where $\chi_{J}(x)$ are the eigenfunctions of the Schrödinger problem

$$
-\chi_{j}^{\prime \prime}(x)+v(x) \chi(x)=\epsilon_{j} \chi_{j}(x)
$$

with boundary conditions $\chi_{j}( \pm l / 2)=0$.

\subsection{Discretization}

The numerical procedure requires the $x$ variable to be discretized as $x_{i} \equiv i h-l / 2$, with $h \equiv l /(N+1)$, for $i=$ $0,1, \ldots, \bar{N}+1$. Equation (13) can then be approximated by the following difference equation:

$$
\begin{gathered}
-\frac{1}{h^{2}}\left[\chi_{J}\left(x_{i+1}\right)-2 \chi_{J}\left(x_{i}\right)+\chi_{J}\left(x_{i-1}\right)\right] \\
+v\left(x_{i}\right) \chi_{j}\left(x_{i}\right)=\epsilon_{j} \chi_{J}\left(x_{i}\right)
\end{gathered}
$$

with boundary conditions $\chi_{j}\left(x_{0}\right)=\chi_{j}\left(x_{N+1}\right)=0$. The problem above is nothing but the diagonalization of the symmetric, tridiagonal $N \times N$ matrix $H$, defined as

$$
H_{i j} \equiv \begin{cases}v\left(x_{l}\right)+2 h^{-2} & \text { if } i=j \\ -h^{-2} & \text { if }|i-j|=1 \\ 0 & \text { otherwise }\end{cases}
$$

$\epsilon_{j}$ being the $j$ th eigenvalue and $\chi_{j}\left(x_{i}\right)(i=1,2, \ldots, N)$ the $i$ th component of the $j$ th eigenvector, with $j$ running from 1 to $N$. The Schrödinger equation is thus transformed into the much simpler problem of diagonalizing $H$, and we can take advantage of its simpler shape.

Once $\epsilon_{j}$ and $\chi_{j}$ are available for $j=1,2, \ldots, N$, the Fermi level, $\epsilon_{F}$, is obtained as the solution of

$$
\left(a^{*}\right)^{2} N_{D}^{+}=\sum_{j=1}^{N} v_{j}
$$

with $v_{j}$ given by $(12 b)$ for every $\epsilon_{F}$. Now the electron density $v\left(x_{i}\right)$ is completely determined via equation (12a) and, accordingly, the full right-hand side of equation (11). Again this latter equation can be approximated by a difference equation, namely

$$
\begin{aligned}
& h^{-2}\left[v_{H}\left(x_{i+1}\right)-2 v_{H}\left(x_{i}\right)+v_{H}\left(x_{i-1}\right)\right] \\
& \quad=8 \pi\left[v_{D}^{3 \bar{D}}\left(x_{i}\right)-v\left(x_{i}\right)\right]
\end{aligned}
$$

with boundary conditions $v_{H}\left(x_{0}\right)=v_{H}\left(x_{N+1}\right)=0$. This problem can be straightforwardly mapped onto that of 
finding the solution to the system $D v_{H}=\rho$, where $D$ is the symmetric, tridiagonal $N \times N$ matrix defined as

$$
D_{i j} \equiv \begin{cases}-2 & \text { if } i=j \\ 1 & \text { if }|i-j|=1 \\ 0 & \text { otherwise }\end{cases}
$$

and $\rho$ is the vector whose components are $\rho_{i} \equiv$ $8 \pi h^{2}\left[v_{D}^{3 D}\left(x_{i}\right)-v\left(x_{i}\right)\right], i=1,2, \ldots, N$. The solution, $v_{H}$, of this linear system can be readily obtained through the standard Thomas algorithm, which amounts to computing the solution iteratively, as follows:

$$
\begin{aligned}
v_{H}\left(x_{N+1}\right) & =0 \\
v_{H}\left(x_{j}\right) & =\alpha_{j} v_{H}\left(x_{j+1}\right)+\gamma_{j}
\end{aligned}
$$

with the index $j$ running backwards $(j=N, N-1, \ldots, 1)$, and where

$$
\begin{array}{cc}
\alpha_{0}=0 & \gamma_{0}=0 \\
\alpha_{j}=\frac{-c_{j}}{d_{j}+a_{j} \alpha_{j-1}} & \gamma_{j}=\frac{\rho_{j}-a_{j} \gamma_{j-1}}{d_{j}+a_{j} \alpha_{j-1}} \\
j=1,2, \ldots, N
\end{array}
$$

$d_{j}(j=1,2, \ldots, N), a_{j}(j=2,3, \ldots, N)$ and $c_{j}$ $(j=1,2, \ldots, N-1)$ are the diagonal, subdiagonal and superdiagonal elements of the matrix $D$ respectively (in our case, $d_{j}=-2$ and $a_{j}=c_{j}=1$ ). Here $\rho_{j}$ is the $j$ th component of the right-hand side vector $\rho$ as defined below equation (18). Note that the undefined elements $a_{1}$ and $c_{N}$ appearing in equation (20) are in fact irrelevant.

\subsection{Algorithm}

The self-consistent algorithm consists of the following steps:

(1) Set $v_{H}\left(x_{i}\right)=v\left(x_{1}\right)=0$ for all $i=1,2, \ldots, N$.

(2) Set $v_{H}^{\text {old }}=v_{H}$.

(3) Compute $v\left(x_{i}\right)$ as given by equation (9).

(4) Diagonalize $H$ (equation (15)) to obtain the eigenvalues, $\epsilon_{j}$, and eigenvectors, $\chi_{j}(j=1.2, \ldots, N)$.

(5) Compute $\epsilon_{F}$ by solving equation (16).

(6) Compute $v\left(x_{i}\right)$ as given by equations (12a,12b).

(7) Determine $v_{H}^{\text {new }}\left(x_{i}\right)$ through the recurrence (19) (notice that the coefficients $\alpha_{j}$ and $\gamma_{j}$ only have to be computed once at the beginning of the program).

(8) Check for convergence (by comparing, for instance, $v_{H}^{\text {new }}$ with $v_{H}^{\text {old }}$ ); if tolerance has been attained then exit the self-consistency process with $v_{H}=v_{H}^{\text {new }}$.

(9) Otherwise set $v_{H}=\lambda v_{H}^{\text {new }}+(1-\lambda) v_{H}^{\text {old }}$ and repeat from step (2) on.

The parameter $\lambda$ has been introduced to control the iteration: if $\lambda=0$ we will have $v_{H}=0$ forever, while if $\lambda=1, v_{H}$ will undergo its maximum variation at every step. This latter case (commonly used throughout the literature) has proven not to converge for $N_{D}^{+} \gtrsim 10^{-12}$ $\mathrm{cm}^{-2}$. Instead, $\lambda=1 / 2$ makes the process convergent for any set of parameters.

The numerical parameters we have been using are as follows. Our mesh consisted of 501 points, enough to resolve the physical dimensions of the delta layer potential we will describe below. We have checked our numerical procedure by using smaller and larger numbers of grid points, finding a negligible dependence on this parameter, as desired. In addition, we verified that the choice of the convergence acceleration parameter $\lambda$ influences only the rate at which the algorithm converges and not the convergence itself, provided it is not too close to 0 or 1 . On the other hand, we usually started the Hartree iteration from $v_{H}(z)=0$; we tested several other choices and we satisfactorily found that the procedure converged always to the same result, thus precluding the possibility of having a fictitious solution. We chose to stop the iterative procedure when the relative variation of the Fermi energy was $\leq 10^{-6}$. We also used another criterion, namely computing the integral of the absolute value of the difference between the new and old potentials and stopping when it was less than $10^{-3}$, obtaining again good agxeement between the two conditions. Typical runs attained convergence after about 20 Hartree iterations, each one of them taking around $30 \mathrm{~s}$ of CPU time on a HP 9000/715/75 with the above integration parameters. This shows that our procedure is rather efficient, and we are confident that it will allow us to deal with the superlattice problem without prohibitive use of CPU time.

\section{Results and discussions}

The different magnitudes we are interested in have been obtained taking $m^{*}=0.067 m_{0}$ and $k=12.7$ in GaAs. Since the dependence of the subband structure upon donor concentration $\left(N_{D}^{*}\right)$, its distribution width $(d)$ and the thickness $(L)$ are well understood in the absence of external fields [19], even if the whole structure is embedded in a quantum well [20], we have fixed their values and concerned ourselves with the dependence on temperature and electric field. In our numerical simulations we have set $N_{D}^{+}=5 \times 10^{12} \mathrm{~cm}^{-2}, d=20 \AA$ and $L=500 \AA$. This choice, with the above numerical parameters, gives our calculation a resolution of $1 \AA$. The maximum electric field we have considered is $F=100 \mathrm{kV} \mathrm{cm}^{-1}$, well below the value for which $\Gamma-\mathrm{X}$ mixing may be observed, thus remaining within the range of validity of our scalar Hamiltonian. We have studied three typical temperatures, namely those of liquid helium, $4.2 \mathrm{~K}$, liquid nitrogen, $77 \mathrm{~K}$, and room temperature, $300 \mathrm{~K}$.

In the absence of an applied electric field, the confining potential presents the characteristic $V$-shape profile, as shown in figure 1 , where the origin of energies of all curves is the Fermi level. On increasing the electric field the potential is tilted so that it becomes slightly asymmetric (see figure 1 for $50 \mathrm{kV} \mathrm{cm}^{-1}$ ). This behaviour is also predicted by means of the Thomas-Fermi approach, as mentioned above [7]. This trend holds until a critical value of the electric field $F_{c}$ (about $60 \mathrm{kV} \mathrm{cm}^{-1}$, see below) is reached. For higher strengths of the electric field, the confining potential changes its shape dramatically and a second minimum appears at the left barrier. Therefore, a local potential barrier arises between the centre and the left barrier of the structure, as shown in figure 1 for 


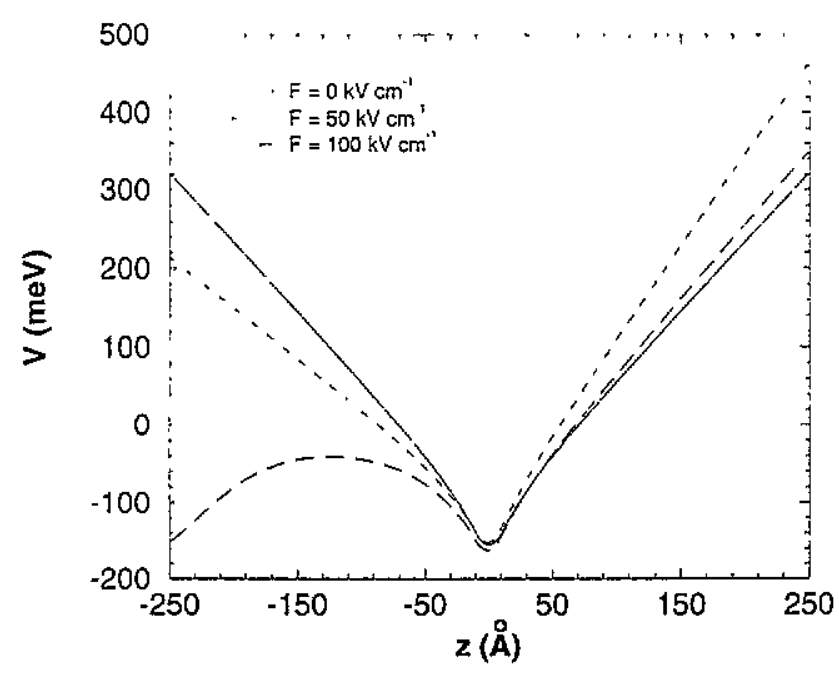

Figure 1. The self-consistent confining potential for an ionized donor concentration $N_{D}^{+}=5 \times 10^{12} \mathrm{~cm}^{-2}$ distributed over $d=20 \dot{A}$ for three different electric fieids, at $T=4.2 \mathrm{~K}$. The zero of energy is set at the Fermi level.

$F=100 \mathrm{kV} \mathrm{cm}^{-1}$. It is worth mentioning that the transition between these two different regimes is very sharp. In other words, $V(-L / 2)$ drops $\sim 300 \mathrm{meV}$ in a very narrow interval, $\leq 1 \mathrm{kV} \mathrm{cm}^{-1}$, of electric fields. This phenomenon can be thought of as arising from competition between opposite effects: on one side, the electric field pushes the charge distribution to the left, and on the other side, electronic repulsion prevents charge accumulation on that part of the system.

The different shapes of the confining potential for $F$ smaller or larger than $F_{c}$ must strongly influence the subband structure and the corresponding subband occupation, as actually occurs. Results at different temperatures are collected in figure 2 . In this figure we have plotted only those subbands whose electron densities $n_{j}$ are at least $1 \%$ of the ground state occupation. As expected, the occupation of subbands above the ground state increases with temperature. The subband energies depend only slightly on the applied electric field up to a critical field $F_{c} \approx 60 \mathrm{kV} \mathrm{cm}^{-1}$, at which a sudden drop of the levels is observed. This value of $F_{c}$ is almost the same for $T=4.2 \mathrm{~K}$ and $T=77 \mathrm{~K}$, whereas at room temperature it is somewhat smaller. It is clear that this drop of the levels is related to the rapid change in the value of $V(-L / 2)$ and the subsequent appearance of the local maximum around $z=-L / 4$. This behaviour is very different to the quantumconfined Stark effect in quantum wells, where subbands change smoothly with $F$ [21].

The subband occupations at three different temperatures and three different values of the electric field are shown in table 1. The occupation of the lowest subband is almost independent of $T$ and $F$. With increasing temperature, more and more subbands are populated, as mentioned above. The most significant feature when increasing the electric field is that the occupation of the excited subbands increases rapidly for fields above $F_{c}$. This fact is remarkable at low temperature, for which the first excited state has an occupation of 0.007 (in units of $10^{12} \mathrm{~cm}^{-2}$ ) for $F<F_{c}$ while it increases by two orders of magnitude
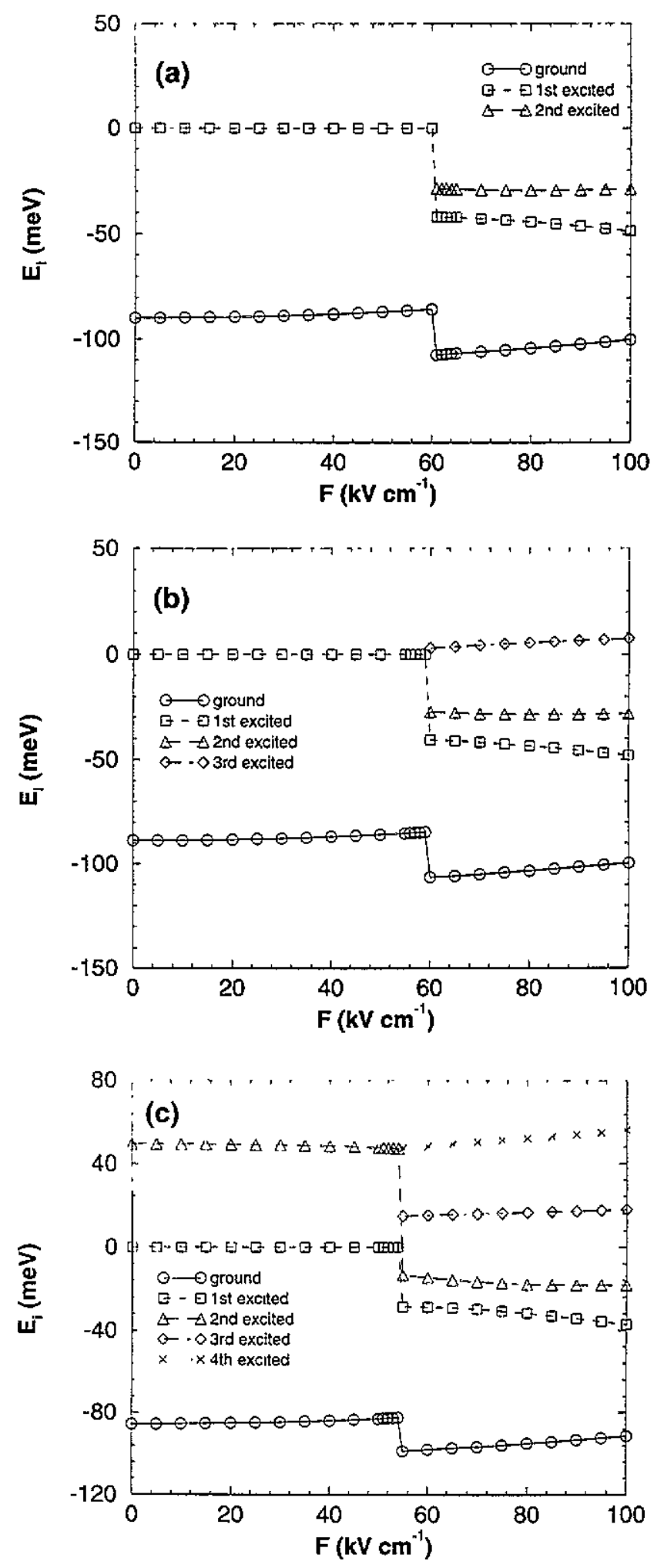

Figure 2. Subband energies as a function of the applied electric field for (a) $T=4.2 \mathrm{~K}$ (b) $T=77 \mathrm{~K}$ and

(c) $T=300 \mathrm{~K}$. Only those subbands with electron densities larger than $1 \%$ of the electron density in the ground state are plotted. Energies are referred to the Fermi level. Note that the vertical scale is different in plot (c) for the sake of clarity.

for $F>F_{c}$. Hence band filling also changes dramatically when crossing $F_{c}$.

According to our previous results, the spatial charge distribution should also be strongly influenced by the 
Table 1. The subband occupation for three different temperatures and electric fields is shown in units of $10^{12} \mathrm{~cm}^{-2}$.

\begin{tabular}{lclllll}
\hline $\begin{array}{l}T \\
(\mathrm{~K})\end{array}$ & $\begin{array}{l}F \\
\left(\mathrm{kV} \mathrm{cm}^{-1}\right)\end{array}$ & Ground & 1st & 2nd & 3rd & 4th \\
\hline 4.2 & 0 & 2.50 & 0.007 & 0 & 0 & 0 \\
& 50 & 2.42 & 0.007 & 0 & 0 & 0 \\
& 100 & 2.80 & 1.36 & 0 & 0 & 0 \\
77 & 0 & 2.48 & 0.13 & 0 & 0 & 0 \\
& 50 & 2.40 & 0.13 & 0 & 0 & 0 \\
& 100 & 2.78 & 1.34 & 0.79 & 0.05 & 0 \\
300 & 0 & 2.42 & 0.50 & 0.10 & 0.02 & 0.005 \\
& 50 & 2.35 & 0.50 & 0.11 & 0.03 & 0.006 \\
& 100 & 2.58 & 1.20 & 0.80 & 0.29 & 0.08 \\
\hline
\end{tabular}

applied electric field. To elucidate these effects, we have calculated the squared envelope functions at low temperature (figure 3). At zero field the envelope functions present a well defined parity since $V(z)$ is an even function; thus the electron density $n(z)$ is symmetric around the $\delta$ doping layer. On increasing the electric field, the expected value $\left\langle\psi_{j}|z| \psi_{j}\right\rangle$ shifts to the left, in the same fashion as in ordinary quantum wells. However, electric fields larger than $F_{c}$ cause the first excited state to be located very close to the left barrier due to the presence of the potential minimum at $z=-L / 2$. Therefore, spatial charge distributions undergo a large shift to the left for $F>F_{c}$, which must clearly have profound effects on intersubband transitions between the first excited subband and the ground subband. This is shown in figure 4 , where $f_{10}$ is plotted as a function of the electric field at three different temperatures. Below $F_{c}$ the oscillator strength is almost constant and presents a high value, close to 15 , which is even larger than that obtained for a narrow $\mathrm{GaAs}-\mathrm{Ga}_{1-x} \mathrm{Al}_{x}$ As quantum well inside a wider one [18]. Thus, for instance, $f_{10}\left(50 \mathrm{kV} \mathrm{cm}^{-1}\right) / f_{10}\left(0 \mathrm{kV} \mathrm{cm}^{-1}\right) \sim$ 0.93 . But contrary to quantum wells, $f_{10}$ decays very fast for electric fields larger than $F_{c}$, so that, for instance, $f_{10}\left(100 \mathrm{kV} \mathrm{cm}^{-1}\right) / f_{10}\left(0 \mathrm{kV} \mathrm{cm}^{-1}\right) \sim 0.1$. This change is practically the same for the two lowest temperatures, the magnitude of the jump becoming smaller at room temperature; nevertheless, the drop of $f_{10}$ is equally abrupt for all temperatures. In closing this section, we note that this drop of the oscillator strength arises from the fact that the secondary well, newly formed, begins to confine charge. As a result, the overlapping between the ground and the first excited states is small, and transitions between the two subbands become tunnelling-like, leading to a sharp decrease of $f_{10}$.

\section{Conclusions}

In the present work we have studied single $\mathrm{Si} \delta$-doped $\mathrm{GaAs}$ under an electric field applied parallel to the growth direction. Electronic structure and intersubband transitions have been calculated by solving the Schrödinger and Poisson equations self-consistently. To this end, we have developed a very efficient numerical code which paves
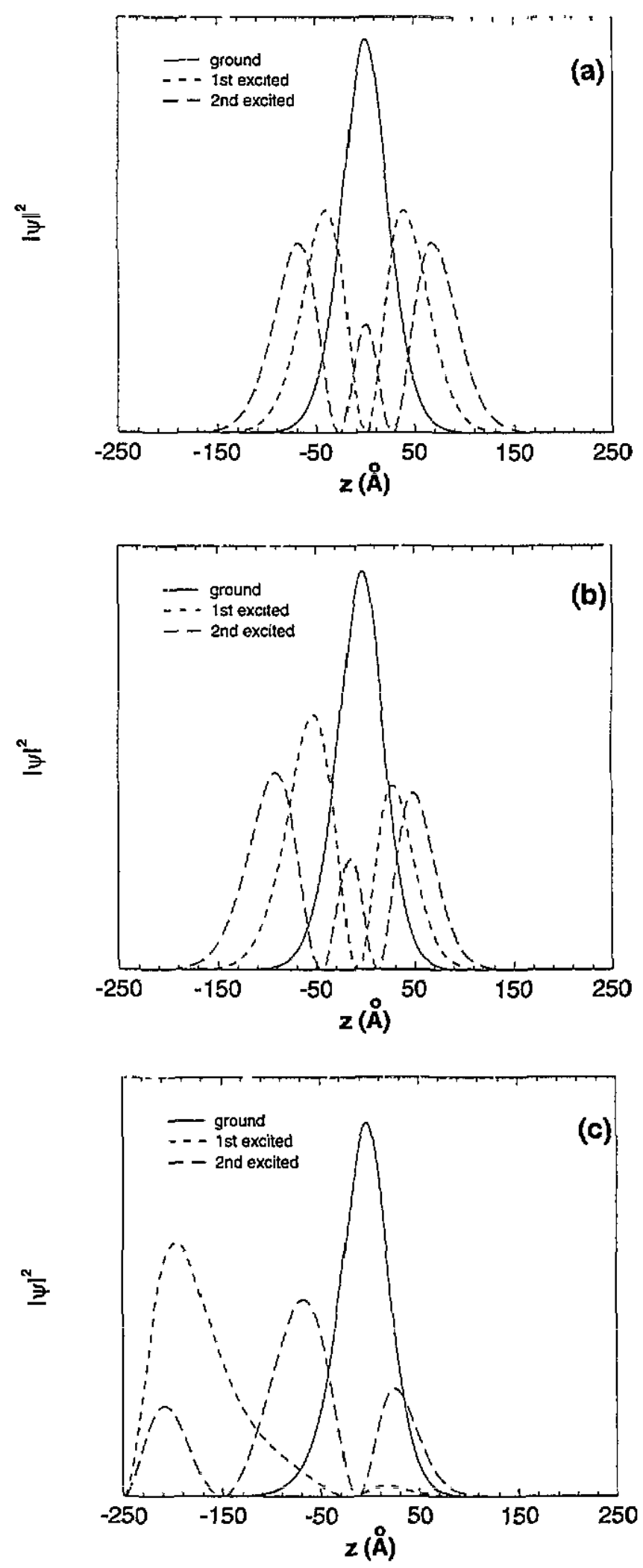

Figure 3. Squared envelope fuctions for the three lowest subbands at different fields: (a) $F=0 \mathrm{kV} \mathrm{cm}^{-1}$,

(b) $F=50 \mathrm{kV} \mathrm{cm}^{-1}$ and (c) $F=100 \mathrm{kV} \mathrm{cm}^{-1}$, at $T=4.2 \mathrm{~K}$.

the way to self-consistent studies of $\delta$-doped superlattices. As regards the specific system we deal with here, one of the most significant results is the existence of a critical value of the electric field $F_{c}$ separating two very different behaviours of the quantum-confined Stark effect, which is very different from what happens in quantum wells. This 


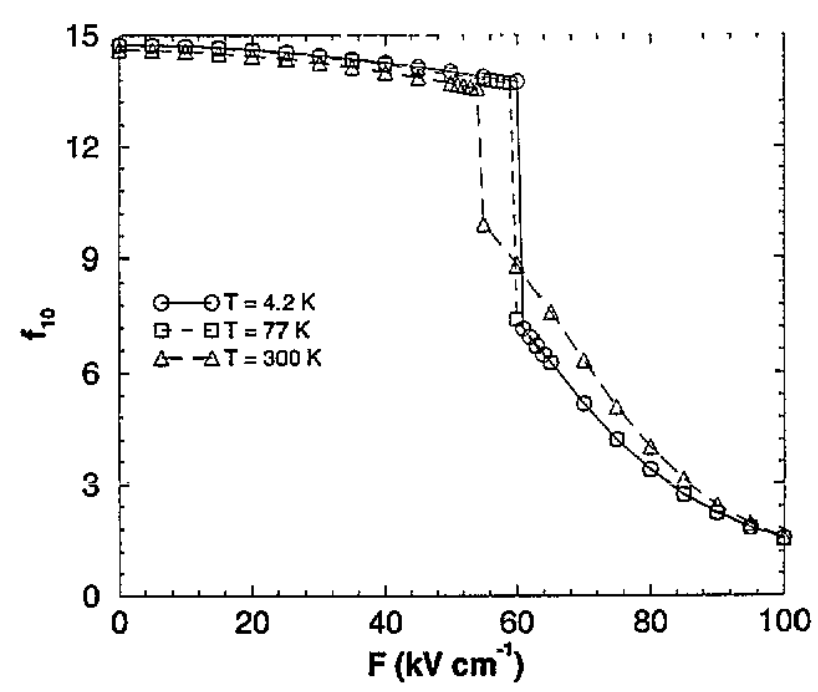

Figure 4. Oscillator strength for the intersubband transition $1 \rightarrow 0$ as a function of the applied electric field at different temperatures.

critical field is related to the fact that the confining potential develops a second minimum at the left barrier. As a consequence, we have observed that the subband energies present a step-like shape as a function of the electric field, with a sharp decrease at $F_{c}$. This is accompanied by a marked increase of the occupation of the first excited subband and a strong shift of the spatial charge distribution. Finally, the oscillator strength for intersubband transitions is very large and almost constant below $F_{c}$ but vanishes quickly above $F_{c}$.

Interestingly, this sharp change in the confining potential shape poses a number of questions about the behaviour of superlattices. From what we now know, it is not clear whether the appearance of an intermediate barrier will help form electric field domains or, on the contrary, will prevent them due to loss of coherence. It is conceivable that electric field domains will appear below $F_{c}$, where the phenomenology is similar to that of quantumwell-based superlattices. The situation is more intriguing above $F_{c}$, because the splitting of the quantum well into two wells might or might not survive in the superlattice. The consequences of the preservation of this effect are difficult to predict, because in that case the superlattice might exhibit a double periodicity or become aperiodic. If double periodicity arises, it could induce unusual electron transport properties, like those predicted for the so-called dimerer superiattices [22]. In addition, it is also evident that the well splitting phenomenon will have consequences in terms of possible resonant tunnelling effects, not only in superlattices but also in double $\delta$-doped layer structures. These and related questions are clearly worth studying, and work along these lines is now in progress [23].

Finally, we want to end the paper with a few comments on the applications of what we have found. The fact that the oscillator strength undergoes a sudden drop allows us to suggest that structures made out of Si $\mathcal{S}$-doped GaAs embedded in a quantum well may be of use as optical filters. Note that dipolar transitions $1 \rightarrow 0$ show a negligible probability above $F_{c}$; as these two subbands contain most of the carriers, this will be the relevant transition governing the optical response of the device. It is important to recall that the value of $F_{c}$ is almost independent of temperature, so that such a device could be used over a wide range of temperatures. In addition, it is clear that the value of $F_{c}$ could be adjusted by changing $N_{D}^{+}, L$ and $d$. Therefore, this kind of structure may open the possibility of fabricating new optoelectronic devices.

\section{Acknowledgments}

JAC thanks partial support from DGICYT (Spain) through project PB91-0378. FD-A and AS are supported from CICYT (Spain) through project MAT95-0325.

\section{References}

[1] Zrenner A, Reisenger H, Koch F and Ploog K 1985 Proc. 17th Int. Conf, on the Physics of Semiconductors (San Francisco 1984) ed J P Chadi and W A Harrison (Berlin: Springer) p 325

[2] Whall T E 1992 Contemp. Phys. 33369

[3] Kortus J and Monecke J 1994 Phys. Rev. B 4917216

[4] İoratti L 1990 Phys. Rev. 4118340

[5] Degani M H 1991 Phys. Rev. 445580

[6] Zrenner A, Koch F and Ploog K 1988 Surf. Sci. 196671

[7] Domínguez-Adame F, Méndez B and Maciá E 1994 Semicond. Sci. Technol. 9263

[8] Domínguez-Adame F and Méndez B 1994 Phys. Rev. B 49 11471

[9] Agulló-Rueda F, Méndez E E and Hong J M 1989 Phys. Rev. B 401357

[10] Grahn H T, Schnejder H and von Klitzing K 1990 Phys. Rev. B 412890

[11] Bonilla L L, Galán, J, Cuesta J A, Martínez F C and Molera J M 1994 Phys. Rev. B 508644

[12] Johnson E A and MacKinnon A 1990 Semicond. Sci. Technol. 5 S189

[13] Hagon J P, Jaros M and Herbert D C 1989 Phys. Rev. B 40 6420

[14] Domínguez-Adame F and Méndez B 1994 Semicond. Sci. Technol. 91358

[15] Hedin L and Lundqvist B I 1971 J. Phys. C: Solid State Phys. 42064

[16] Harwit A and Harris J S 1987 Appl. Phys. Lett. 50685

[17] Yuh P F and Wang K L 1989 IEEE J. Quantum Electron. 251671

[18] Chen W Q and Andersson T G 1993 J. Appl. Phys. 734484

[19] Degani M H 1991 J. Appl. Phys. 704362

[20] Xu W and Mahanty J 1994 J. Phys.: Condens. Matter 6 4745

[21] Matsuura M and Kamizato T 1986 Phys. Rev. B 338385

[22] Diez E, Sánchez A and Domínguez-Adame F 1994 Phys. Rev. B 5014359

Domínguez-Adame F, Sánchez A and Diez E 1994 Phys. Rev. B $\mathbf{5 0} 17736$

[23] Cuesta J A, Sánchez A and Domínguez-Adame F 1994 unpublished 